\title{
Occurrence and profiles of bisphenol analogues in municipal sewage sludge in China
}

\author{
Shanjun Song, Maoyong Song, Luzhe Zeng, Thanh Wang, Runzeng Liu, Ting Ruan*, \\ Guibin Jiang \\ State Key Laboratory of Environmental Chemistry and Ecotoxicology, Research Center for Eco-Environmental Sciences, Chinese Academy of Sciences, Beijing \\ 100085, China
}

\section{A R T I C L E I N F O}

\section{Article history:}

Received 13 September 2013

Received in revised form

22 November 2013

Accepted 26 November 2013

\section{Keywords:}

Bisphenol analogues

Composition profile

Sewage sludge

Estrogenic equivalent

\begin{abstract}
A B S T R A C T
Extensive use of bisphenol A and its analogues has caused increasing concern over the potential adverse health impacts of these chemicals. In this study, the presence and profiles of 13 bisphenols (BPs) were investigated in 52 municipal sewage sludge samples collected from 30 cities in China. Tetrabromobisphenol A was the most frequently observed analogue (geometric mean: $20.5 \mathrm{ng} / \mathrm{g} \mathrm{dw}$ ). Bisphenol A (4.69 ng/g dw), bisphenol S (3.02 ng/g dw), and bisphenol F (3.84 ng/g dw) were found with similar frequency. Other BP analogues such as tetrachlorobisphenol $\mathrm{A}$, bisphenol $\mathrm{AF}$, bisphenol $\mathrm{E}$, and dihydroxybiphenyl were identified for the first time in sewage sludge in China. Significant correlations were found among BP concentrations, but no relationships were found with wastewater treatment plant characteristics. Profiles of the relative estradiol equivalents suggested that the estrogenic potential of BP mixtures may be associated with the occurrence and contributions of specific analogues.
\end{abstract}

(c) 2013 Elsevier Ltd. All rights reserved.

\section{Introduction}

Bisphenols (BPs) are a large group of anthropogenic chemicals that share similar molecular structures containing two parahydroxyphenyl functionalities. BPs are widely used as additives and/or reactive raw materials in various industrial and commercial applications such as fire-resistant polymers, plastic linings for food containers, dentistry sealants, and thermo-sensitive coatings for paper materials (Delfosse et al., 2012).

Owing to potential endocrine disrupting properties and ecological impacts, there has been increasing concern regarding production and use of BP homologues, such as bisphenol A (BPA) and variants (e.g., bisphenol S (BPS) and bisphenol F (BPF)) (Liao et al., 2012a, 2012b). However, very limited data are currently available on the presence and distribution of BP analogues in the environment. BPS, $\mathrm{BPF}$, and other BP analogues such as bisphenol B (BPB) have been detected in beverages and canned foods, although their detection frequencies and concentrations were several times lower than those of BPA (Cacho et al., 2012; Cunha et al., 2011; Vinas et al., 2010). BPS and BPB have also been found in human blood sera and urine at part per billion levels (Cobellis et al., 2009; Cunha and Fernandes, 2010).

\footnotetext{
* Corresponding author.

E-mail address: tingruan@rcees.ac.cn (T. Ruan).
}

In the environment, BPF was first reported in surface water, sewage, and sediments in Germany (Fromme et al., 2002). BPA, BPS, and BPF were recently found to be the predominant BP analogues in sediments and indoor dust samples obtained from North America and several Asian countries, while bisphenol AF (BPAF), BPB, and bisphenol AP (BPAP) were only infrequently detected (Liao et al., 2012c, 2012d). Halo-substituted BP flame retardants such as tetrabromobisphenol A (TBBPA) have also been frequently detected in soil, sediments, indoor dust, and biota due to extensive use in various polymers (He et al., 2010; Nyholm et al., 2013).

The human health effects of BPA have been well-studied; relationships have been found between BPA exposure and adverse effects on physiological functions including neural and behavioral dysfunction (Yeo et al., 2013) and reproductive effects (Li et al., 2010). Due to their similar molecular structures and physicochemical properties, other BP analogues may also exhibit similar toxicological effects. However, individual BPs showed varied estrogenicity properties, which were elucidated as discrete binding modes between BP analogues and corresponding nuclear hormone receptors (Delfosse et al., 2012).

Because wastewater treatment plants (WWTPs) may be significant sources of BPs to the environment such as in land application of biosolids, identification and quantification of these pollutants in digested sludge provides information for risk assessment for the 
surrounding environment (Clarke and Smith, 2011). In the present study, municipal sewage sludge from various Chinese cities was collected and analyzed to provide information on the contamination levels, distributions, and relative estrogenic activity potentials of BPA and ascendant alternatives (BPA, BPS and BPF), halosubstituted BP flame retardants (TBBPA and TCBPA) and other BP analogues in the sludge.

\section{Materials and methods}

\subsection{Materials}

The chemical names, abbreviations, and structures of the chemicals included in this study are shown in Fig. S1. BPA, BPB, bisphenol C (BPC), bisphenol E (BPE), BPF, BPS, BPAF, BPAP, 2,4-dihydroxybenzophenone (DHBP), dihydroxydiphenyl sulfide (TDP), tetramethylbisphenol A (TMBPA), TBBPA, and tetrachlorobisphenol A (TCBPA) were obtained from TCI (Tokyo, Japan). Mass-labeled ${ }^{13} \mathrm{C}$-BPA (RING- ${ }^{13} \mathrm{C}_{12}, 99 \%$ ) and ${ }^{13} \mathrm{C}$-TBBPA (RING $-{ }^{13} \mathrm{C}_{12}, 99 \%$ ) were obtained from Cambridge Isotope Laboratories (Andover, MA). All chemicals had a purity of $\geq 98 \%$ unless otherwise stated. HPLCgrade methanol and tetrahydrofuran was supplied by J.T. Baker (Phillipsburg, NJ). Ultrapure water (18.3 M $\Omega$ ) was generated using a Milli-Q system (Millipore, Billerica, MA). ENVI-Carb $(0.5 \mathrm{~g}, 6 \mathrm{~mL})$ and Sep-Pak C18 $(1 \mathrm{~g}, 6 \mathrm{~mL})$ solid-phase extraction cartridges were obtained from Supelco (St. Louis, MO) and Waters (Milford, MA), respectively.

\subsection{Sample collection}

Details of sample collection can be found in Ruan et al. (2012). In brief, one grab sample from each of 52 municipal WWTPs in 30 cities in China was collected during October 2010-May 2011. Samples were collected from 20 provinces and municipalities, most of which were located in more developed regions. Approximately $500 \mathrm{~g}$ of freshly digested sludge samples were collected at the dehydration process, packed in aluminum foil, sealed in polypropylene bags, and then immediately delivered to our laboratory. The samples were freeze-dried, homogenized, and held at $-20{ }^{\circ} \mathrm{C}$ until analysis. Detailed information associated with each sludge sample such as the sampling locations, WWTP capacity, type of treatment, and sludge characteristics is provided in Supplementary Data (Table S1).

\subsection{Sample pretreatment and analysis}

Sludge extraction and cleanup procedures were as previously reported with minor modifications (Liao et al., 2012c). Briefly, $\sim 0.3 \mathrm{~g}$ of each sample was placed in a $15-\mathrm{mL}$ polyethylene terephthalate centrifuge tube (Corning Inc., Corning, NY) and extracted with $5 \mathrm{~mL}$ of methanol by shaking at $350 \mathrm{rpm}$ for $60 \mathrm{~min}$. The sample was spiked with $100 \mathrm{ng}{ }^{13} \mathrm{C}_{12}$-TBBPA internal standard (IS) prior to extraction. The supernatant was collected after centrifugation at $4800 \mathrm{rpm}$ for $10 \mathrm{~min}$. Extraction was repeated three times and the supernatants were combined and then passed through a 6-mL methanol preconditioned ENVI-Carb cartridge to eliminate matrix interferences (Pang et al., 2013). Analyte residues on the ENVI-Carb cartridge were then eluted with $3 \times 2 \mathrm{~mL}$ methanol and $3 \times 2 \mathrm{~mL}$ tetrahydrofuran/methanol $(4: 6, \mathrm{v})$ v) mixture, successively. All eluates were combined and concentrated to $0.5-1 \mathrm{~mL}$ under a gentle nitrogen stream at room temperature, diluted with $15 \mathrm{~mL}$ ultrapure water, and loaded onto a Sep-Pak C18 cartridge for further purification. The C18 cartridge was preconditioned with $6 \mathrm{~mL}$ methanol and then with $6 \mathrm{~mL}$ ultrapure water. After sample loading, the cartridge was washed with $6 \mathrm{~mL}$ ultrapure water and the analytes were then eluted with $3 \mathrm{~mL}$ methanol in triplicate and finally concentrated to $1 \mathrm{~mL}$ under a nitrogen stream. One hundred nanograms of ${ }^{13} \mathrm{C}_{12}$-BPA were then spiked into each sample vial before instrument analysis.

A Quattro Premier XE triple-quadrupole mass spectrometer coupled with an Alliance 2695 high-performance liquid chromatography (HPLC-MS/MS; Waters Inc., Milford, MA) was used for quantification. Twenty microliters of the sample extract were injected into a Symmetry Shield C18 analytical column $(2.1 \mathrm{~mm}$ i.d. $\times 150 \mathrm{~mm}$ long, $5 \mu \mathrm{m}$; Waters) for analyte separation. The column temperature was $40{ }^{\circ} \mathrm{C}$ and the flow rate was $0.3 \mathrm{~mL} / \mathrm{min}$. The flow gradient was initiated at a composition of $1: 9$ (methanol/water, v/v), linearly increased to $100 \%$ methanol over $12 \mathrm{~min}$, and held for another $3.5 \mathrm{~min}$. Electrospray ionization (ESI) was operated in the negative mode with a capillary voltage of $3.0 \mathrm{kV}$. The ionization source and desolvation temperatures were optimized at 120 and $320^{\circ} \mathrm{C}$, respectively. Detailed multiple reaction monitoring (MRM) parameters for each of the analytes are given in the Supplementary Data (Table S2). The experimental procedures for BP estrogenic activity analysis using a MVLN estrogen luciferase reporter assay and related results (Fig. S2 and Tables S4 and S5) are also summarized in the Supplementary Data.

\subsection{Quality assurance/quality control}

For positive identification and quantification of the analytes, the confirmation criteria of Liao et al. (2012c) were used with minor modifications. The supernatants from the third extraction of 10 randomly selected sludge samples were pretreated and analyzed separately from those of the first two extraction procedures. Only about $5 \%$ of the BPA and $1 \%$ of the TBBPA remained in the third extract, suggesting that three extraction cycles were sufficient. The recoveries for the matrix-spiked samples (mean \pm standard deviation, Table S6) ranged from $62 \pm 3 \%$ (TDP) to $106 \pm 12 \%$ (TBBPA) for 10 -ng spiked samples and $65 \pm 2 \%$ (TDP) to $118 \pm 14 \%$ (TMBPA) for 100-ng spiked samples, with means of $81 \%$ (10 $\mathrm{ng}$ ) and $88 \%$ (100 ng), respectively. Recoveries for matrix-matched spikes of IS (100 ng, ${ }^{13} \mathrm{C}_{12}$-TBBPA) in field samples were $85 \pm 10 \%$ and matrix interference in the instrument analysis of ${ }^{13} \mathrm{C}_{12}$-BPA was $96 \pm 17 \%$.

Quantification was based on an external calibration method and was corrected based on the IS recoveries $\left({ }^{13} \mathrm{C}_{12}\right.$-TBBPA). Two laboratory blanks of $0.3 \mathrm{~g}$ solventwashed diatomaceous earth (Dionex, Sunnyvale, CA) were included with each batch of 6 samples. Most analytes in the blanks were undetected except for BPA $(\sim 0.10 \mathrm{ng} / \mathrm{mL})$, which was present at $<15 \%$ of the concentrations in the samples. The analyte concentrations were therefore not corrected for blank contamination. The method quantification limit (MQL), calculated as a signal-to-noise ratio of 10 using the 10-ng matrix-spiked samples, ranged from 0.08 (BPE) to 12.8 (TMBPA) ng/g sludge (dry weight, dw). The linear dynamic range of the instrument response $\left(r^{2}>0.99\right)$ was certified daily using calibration standards with concentrations of $1-$ $200 \mathrm{ng} / \mathrm{mL}$.

\subsection{Statistical analysis}

Geometric mean (GM), median, and concentration ranges were used to describe the results. Non-quantified BPs with a signal-to-noise ratio $<10$ in each sludge sample were assigned a value equal to the MQL divided by the square root of 2 . All data were log-transformed for statistical analyses. Spearman's correlation and oneway ANOVA analysis with the Tukey test were conducted using SPSS V17.0 for Windows (SPSS Inc., Chicago, IL). Significance was determined at $p<0.05$ unless otherwise stated.

\section{Results and discussion}

\subsection{Occurrence of bisphenol analogues in sludge}

Concentration distributions, composition profiles, and descriptive statistics for the various BPs in sewage sludge are summarized in Table 1 and Fig. 1. All data are reported on a dry weight (dw) basis. Among the target analytes, 8 BP analogues, TBBPA, TCBPA, BPA, BPS, BPF, BPE, BPAF and DHBP, were positively identified and quantified in the sewage sludge samples with total BP concentrations (sum of bisphenols: $\Sigma$ BPs) of $8.98-600 \mathrm{ng} / \mathrm{g}$.

\subsection{Analogue concentrations and composition profiles}

TBBPA was the dominant BP analogue (GM: $20.5 \mathrm{ng} / \mathrm{g}$, median: $24.7 \mathrm{ng} / \mathrm{g}$ ), making up 3.63-83.1\% (mean 37.4\%) of the total BP residues. This was successively followed by BPA (GM: 4.69, median: $9.38 \mathrm{ng} / \mathrm{g})$, BPS $(3.02,4.34 \mathrm{ng} / \mathrm{g})$, and BPF $(3.84,1.97 \mathrm{ng} / \mathrm{g})$ with mean contributions of $19.9,15.7$, and $11.5 \%$, respectively. BPE $(0.23$, $0.06 \mathrm{ng} / \mathrm{g}), \mathrm{TCBPA}(0.76,0.29 \mathrm{ng} / \mathrm{g})$, DHBP $(0.72,0.20 \mathrm{ng} / \mathrm{g})$, and $\operatorname{BPAF}(0.85,0.42 \mathrm{ng} / \mathrm{g})$ were also detected in the sludge samples with low detection frequencies (mean: $32.7 \%$ (BPE) to $57.7 \%$ (TCBPA)), and contributed about $6.0,3.7,3.3$, and $2.4 \%$ of the total, respectively. However, the other cresol- (BPC, TMBPA) and sulfidesubstituted (TDP) analogues, as well as BPB and BPAP, were not detected in any sample.

The relatively high levels of TBBPA in sewage sludge is likely associated with its increasing industrial application as a brominated flame retardant after phase-out of the commercial pentaand octabrominated diphenyl ethers (Zota et al., 2013). There have been a few studies reporting concentrations of TBBPA in sewage sludge, mainly from Europe and North America. Lee and Peart (2002) reported concentrations of TBBPA ranging over 1-46.2 ng/ $\mathrm{g} \mathrm{dw}$ in 34 sewage sludge samples collected from cities across Canada, and studies have also reported TBBPA at concentrations of 2.1-28.3 in Ontario (Chu et al., 2005) and $300 \mathrm{ng} / \mathrm{g} \mathrm{dw}$ in the Montreal area (Saint-Louis and Pelletier, 2004). Similar levels of TBBPA residues in WWTP sludge were also found in the Netherlands (2-600 ng/g dw; Morris et al., 2004), United Kingdom (15.9-112 ng/g dw; Morris et al., 2004), Sweden ( $<0.3-220 \mathrm{ng} / \mathrm{g}$ wet weight; Oberg et al., 2002), and Spain ( $<3-472$ ng/g dw; Gorga 
Table 1

Descriptive statistics of identified BPs (ng/g d.w.) in the investigated municipal sewage sludge samples from China $(n=52)$.

\begin{tabular}{|c|c|c|c|c|c|c|c|c|c|}
\hline & BPA & BPS & $\mathrm{BPF}$ & ТВBPA & TCBPA & $\mathrm{BPE}$ & BPAF & DHBP & $\Sigma$ BPs \\
\hline Geometric mean & 4.69 & 3.02 & 3.84 & 20.5 & 0.76 & 0.23 & 0.85 & 0.72 & 74.5 \\
\hline Median & 9.38 & 4.34 & 1.97 & 24.7 & $0.29^{c}$ & $0.06^{c}$ & $0.42^{\mathrm{c}}$ & $0.20^{c}$ & 101 \\
\hline Range & $0.42^{c}-152$ & $0.17^{c}-110$ & $1.57^{\mathrm{c}}-143$ & $0.95^{c}-259$ & $0.29^{c}-143$ & $0.06^{c}-167$ & $0.42^{c}-45.1$ & $0.20^{c}-110$ & $5.37-599$ \\
\hline Detection ratio ${ }^{\mathrm{a}}(\%)$ & 76.9 & 82.7 & 63.5 & 96.2 & 57.7 & 32.7 & 46.2 & 55.8 & 100 \\
\hline Quantification ratio ${ }^{\mathrm{b}}(\%)$ & 59.6 & 73.1 & 50.0 & 84.6 & 36.5 & 23.1 & 42.3 & 40.4 & 100 \\
\hline Averaged composition (\%) & 19.9 & 15.7 & 11.5 & 37.4 & 3.7 & 6.0 & 2.4 & 3.3 & 100 \\
\hline
\end{tabular}

a Detection ratio represents the percentage of identified BP analogue with signal/noise $\geq 3$ in the whole batch of samples.

b Quantification ratio represents the percentage of quantified BP analogue with signal/noise $\geq 10$ in the whole batch of samples.

c Filled data as MQL divided by the square root of 2 for non-quantified instrument responses of BPs with the signal/noise ratio below 10 .

$20 \mathrm{ng} / \mathrm{g}$ d.w. (BPA and ascendent alternatives)

$20 \mathrm{ng} / \mathrm{g}$ d.w. (Halo-substituded BP flame retardants)

20 ng/g d.w. (Other BP analogues)

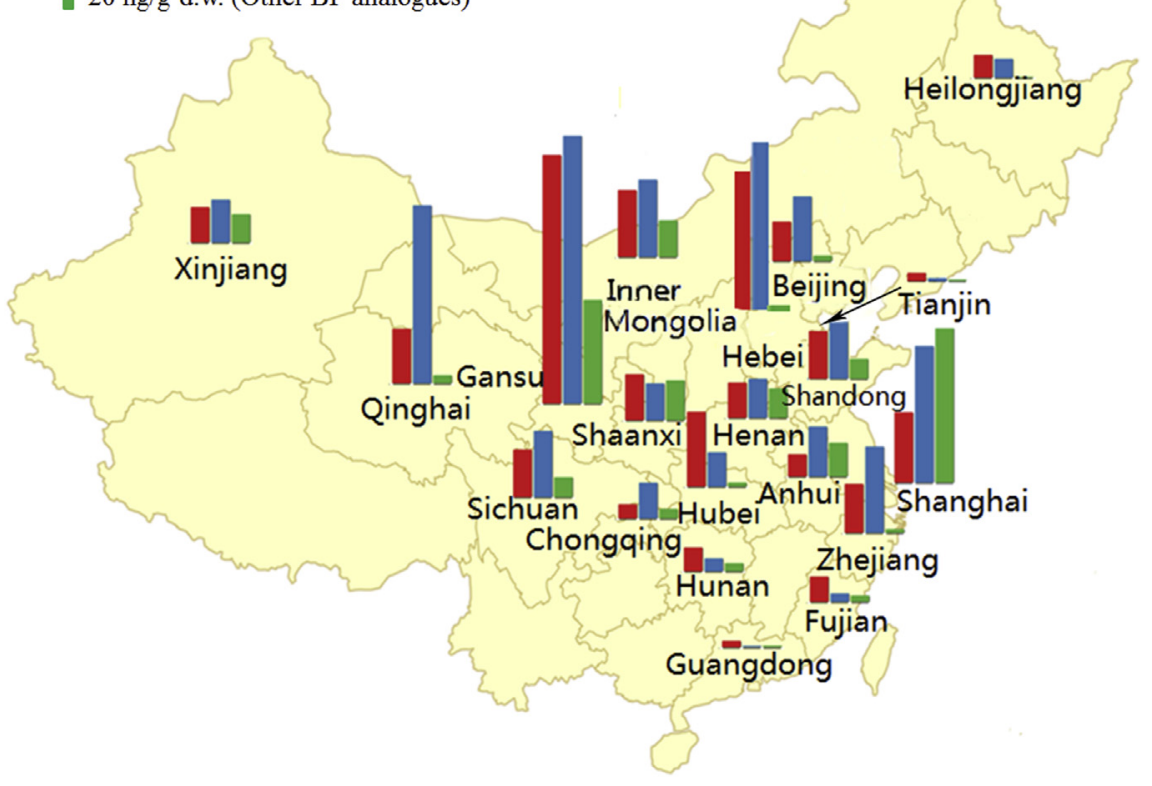

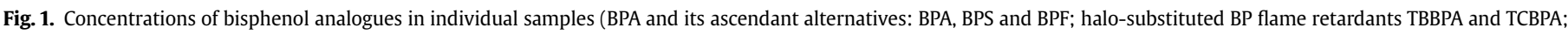
and other BP analogues BPAF, DHBP, and BPE).

et al., 2013). The TBBPA concentrations found in the present study $(<0.4-259 \mathrm{ng} / \mathrm{g}$, with a detection frequency of $96.2 \%)$ are comparable to those published previously and further suggest the widespread use and distribution of this chemical in China. Reports on the presence and distribution of TCBPA in the environment are few, likely due to its limited industrial use. TCBPA has been found in wastewater treatment sludge and pollution control plants at 0.14$0.54 \mathrm{ng} / \mathrm{g} \mathrm{dw}$ in Canada (Chu et al., 2005), and at $\sim 1.4 \mu \mathrm{g} / \mathrm{L}$ in final effluent from wastepaper recycling plants in Japan (Fukazawa et al., 2001). Trace amounts of TCBPA have also been detected in human plasma in Norway (Thomsen et al., 2001). The detected TCBPA residues in this study were $<0.13-143 \mathrm{ng} / \mathrm{g}$ with a median concentration of $0.29 \mathrm{ng} / \mathrm{g}$. The high detection frequency of $57.7 \%$ may reflect wide-spread use of TCBPA as a flame retardant and an epoxy intermediate in China. This finding should be followed up with studies of its occurrence in other environmental media and potential exposure risks due to its estrogenic nature and adverse effects on the thyroid (Kitamura et al., 2005).

Detailed information on BPA analogues in environmental matrices is also scarce. Liao et al. (2012c, 2012d) investigated BPs in indoor dust and sediments from industrialized areas in the United States and several Asian countries. BPA, BPS, and BPF were the predominant analogues, constituting $\sim 78.8-100 \%$ of total BPs.
Elevated concentrations of BPS were also found in daily-use paper products and urine samples, indicating human exposure to BPS (Liao et al., 2012a, 2012b). This may be due to government-wide efforts since the 2000s to eliminate use of BPA by developing alternative chemicals such as BPS for commercial applications such as thermal receipts (Liao et al., 2012c). In the present study, BPA, BPS, and BPF had mean contributions of $34.9,29.7$, and $18.9 \%$ to the total, respectively, and together, constituted $83.6 \%$ of total BPs, excluding the halo-substituted BP flame retardants (TBBPA and TCBPA) (Fig. 2). The BP concentration profiles in the present study were consistent with those for indoor dust samples from China (Liao et al., 2012d), which may imply that sewage sludge is an appropriate sampling medium for environment monitoring of $\mathrm{BP}$ analogues.

Detection of additional BPs in sewage sludge in the present study is also interesting. BPAF was frequently detected in indoor dust and sediment samples in Korea and also sporadically in Japan (Liao et al., 2012d). However, it was not detected in the United States or China (Liao et al., 2012c, 2012d). Recently, a study indicated that manufacturing of polycarbonate copolymers containing BPAF for electronic materials, gas-permeable membranes, and other applications in China could impact the surrounding environment and nearby residents (Song et al., 2012). In the present 

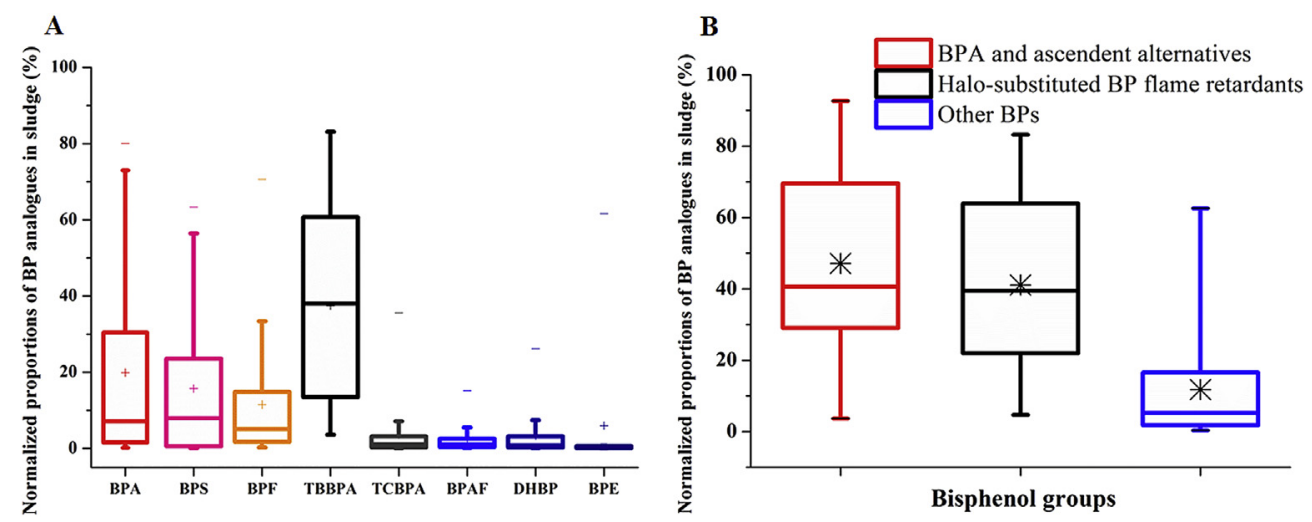

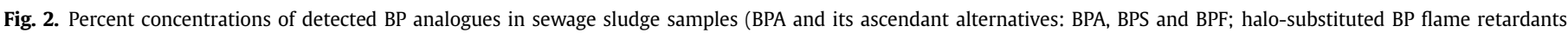

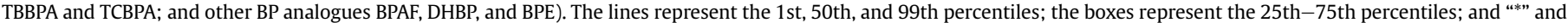
“-” represent the arithmetic mean and maximum/minimum percent of the BP in the entire population of samples, respectively.

study, BPAF was found in nearly half of the samples (24/52) with a maximum concentration of $45.1 \mathrm{ng} / \mathrm{g} \mathrm{dw}$, further confirming the presence of BPAF in certain consumer products. DHBP and BPE, which are crosslinking materials in the synthesis of polyarylate copolymers (Wright and Paul, 1997), were also observed with a detection frequency of 55.8 and $32.7 \%$ in our sludge samples, respectively. Currently, little information is available concerning their presence and behavior in environment matrices and biota. More routine analysis for these two BPs in environmental samples is warranted, as in vitro toxicological data indicate that DHBP and BPE have similar endocrine-disrupting potential to BPA (Chen et al., 2002; Kitamura et al., 2005).

Overall, BPA and its ascendant alternatives (BPS and BPF) and halo-substituted flame retardants (TBBPA and TCBPA) were the two predominant groups of detected BP analogues with mean contributions of $47.1 \%$ and $41.1 \%$ of the total, respectively. The other detected BP analogues (BPE, BPAF, and DHBP) accounted for only a small percentage (11.8\%) of the total (Fig. $2 \mathrm{~B}$ ).

\subsection{Correlations between bisphenol analogues and influencing factors}

Detailed information on the spatial distributions of the BP analogues is summarized in Table S1. No obvious geographic trends were found for most BP analogues. However, extreme values were observed for BPs such as BPE and BPF, which may indicate centralized manufacturing/usage at certain sampling locations. As shown in Table 2, the detected TBBPA concentrations were significantly correlated with those of BPA $(r=0.227, p<0.05)$, BPS $(r=0.303, p<0.05)$, and DHBP $(r=0.439, p<0.01)$ and a similar relationship was found between TCBPA and BPA $(r=0.459$, $p<0.01$ ). However, no such positive relationships were obtained among BPA, BPS, and BPF, which may imply discrete use of these analogues. No correlations (one-way ANOVA, $p>0.05$ ) were observed between BP concentrations and total organic carbon (TOC) content in sludge samples, differing from previously reported results for other hydrophobic phenolic contaminants, such as hydroxylated polybrominated diphenyl ethers (Sun et al., 2013) and benzotriazole ultraviolet stabilizers (Ruan et al., 2012), in the same sewage sludge samples.

Although various BP sources, manufacturing, and applications occur in different regions, the observed trends for BP analogues in WWTPs can partially be explained by their physicochemical properties. Parameters calculated using a screening-level quantitative structure-property relationship model (QSPR; EPI Suite v.
4.11, US EPA) are shown in Table S3. Except for the halogenated derivatives (TBBPA, TCBPA, and BPAF), the octanol-water partition coefficient ( $\log \mathrm{K}_{\mathrm{ow}}$ ) of most BP analogues ranges from 1.65 (BPS) to 3.64 (BPA), suggesting that adsorption to sludge may not be the primary fate of these chemicals in WWTP processes. Rapid physical sorption of BPA onto activated sludge was observed to be the initial removal process in a laboratory-scale batch experiment (Zhao et al., 2008). However, variations in operating conditions such as temperature (Zhao et al., 2008), pH (Cirja et al., 2008), and mixed liquid suspended solid content (Zhao et al., 2008) can result in varying adsorption removal efficiencies. He et al. (2013) reported that BPA could be efficiently removed in chemical/biological processes, indicating that biodegradation/biotransformation were major removal mechanisms. Cases et al. (2011) compared various wastewater treatment processes (conventional activated sludge and membrane bioreactors) for BPA elimination and found that similar efficiencies could be achieved; in the present study, no correlation was found between specific WWTP treatment processes (Table S1) and log-transformed BP concentrations. The environmental fate of BPs may also be associated with biodegradation potential. BIOWIN3 in EPI Suite is a biodegradation model that is frequently used to estimate the degradation half-lives of organic chemicals. BIOWIN3 scores for selected BPs ranged from 2.82 (DHBP) to 2.60 (BPA), indicating that the expected total degradation time was on the order of "weeks" to "weeks-months" for these compounds (Table S3). STPWIN32 (EPI Suite, default sewage treatment plant system properties, BIOWIN output, and EPA draft function for assigning half-lives) was used to simulate the effect of degradation half-lives in the WWTP treatment processes (Table S3). The results clearly indicated that biodegradation (29.9-77.9\% removal) may be a more important elimination mechanism than adsorption (0.77.9\% removal) for BPA, BPS, BPE, BPF, and DHBP.

Table 2

Spearman's correlation coefficients for the detected BP concentrations.

\begin{tabular}{lcrlll}
\hline & BPA & BPS & BPF & TBBPA & TCBPA \\
\hline BPS & -0.036 & & & & \\
BPF & 0.090 & $-0.373^{\mathrm{b}}$ & & & \\
TBBPA & $0.277^{\mathrm{c}}$ & $0.303^{\mathrm{c}}$ & 0.056 & & \\
TCBPA & $0.459^{\mathrm{b}}$ & -0.201 & 0.267 & 0.074 & \\
DHBP & -0.143 & 0.272 & 0.087 & $0.439^{\mathrm{b}}$ & -0.224 \\
\hline
\end{tabular}

a Concentration correlation analysis was performed among identified BPs with detection ratio above $50 \%$ after log-transformed.

b Represents significant correlations at $p<0.01$ level.

${ }^{c}$ Represents significant correlations at $p<0.05$ level. 


\subsection{Relative estrogenic activity potential profiles for bisphenol analogues}

Several studies have indicated potential associations between exposure to BPA and increased cancer risks (Konkel, 2013; Soto et al., 2013). Other BP analogues (e.g., BPF and BPS) are also considered estrogenic (Chen et al., 2002); the estrogenic activities of the BP analogues have been systematically studied in detail (Kitamura et al., 2005). Kitamura et al. (2005) reported relatively higher potencies for TCBPA, BPAF, and BPA in an MCF-7 E-screen in vitro assay, but that of TBBPA was hundreds of times lower. BPS, BPE, and BPF showed estrogenic activity potentials in the MCF-7 assay (Kitamura et al., 2005), as did BPF and DHBP (Kanai et al., 2001). The concentration-response relationships for our investigated BPs were also confirmed using a similar MCF-7 estrogen luciferase reporter assay (MVLN cell line), summarized in the Supplementary Data. The obtained BP half maximal effective concentrations (EC50) were nearly consistent with the previously reported relative estrogenic activities. The observed individual estradiol equivalency factors (EEFs) for BPF, BPAF, BPE, and BPS were $11.6-66.3 \times 10^{-6}$, with relative response factors ranging from 0.22 to 1.93 (Table S4). These results are comparable with previously published values, demonstrating consistency among various experimental approaches. Based on the quantified BP residue concentrations and concentration-response relationships in the in vitro assays, profiles of relative estrogenic activity potentials for the $\mathrm{BP}$ analogues in the sludge samples were preliminarily determined. The percent $17 \beta$-estradiol equivalent $\left(E_{2} E Q \%\right)$ contributed by each analogue per sample can be estimated using the individual EEF multiplied by the observed BP concentrations:

$\mathrm{EEF}_{\mathrm{i}}=\mathrm{EC} 50\left[\mathrm{E}_{2}\right] / \mathrm{EC} 50\left[\mathrm{BP}_{\mathrm{i}}\right]$

$\mathrm{E}_{2} \mathrm{EQ}_{\mathrm{i}} \%=\mathrm{EEF}_{\mathrm{i}} \times \mathrm{Cs}_{\mathrm{i}} / \sum \mathrm{E}_{2} \mathrm{EQ}_{\mathrm{i}}$

where $\mathrm{EC} 50\left[\mathrm{E}_{2}\right]$ and $\mathrm{EC} 50\left[\mathrm{BP}_{\mathrm{i}}\right]$ are the half maximal effective concentrations $(\mu \mathrm{M})$ of $E_{2}$ and the individual BP analogue, respectively, calculated from the concentration-response curves from the luciferase reporter assay (Fig. S2 and Table S4). $\mathrm{Cs}_{\mathrm{i}}$ is the observed BP concentration in the sludge samples ( $\mathrm{ng} / \mathrm{g} \mathrm{dw}$ ). To avoid overestimating the estrogenic activity potentials, only quantifiable BP concentrations were included. Overall, the total $17 \beta$-estradiol equivalents for the BPs in the sludge samples were $0.06-63.9 \mathrm{pg} / \mathrm{g}$ $\mathrm{E}_{2} \mathrm{EQ} \mathrm{dw}$. Thus, minor estrogenic activity may be induced by these $\mathrm{BP}$ analogues, as $\mathrm{E}_{2}$ levels have been reported as several $\mathrm{ng} / \mathrm{g} \mathrm{dw}$ in sludge in China (Liu et al., 2011), hundreds of times higher than those of the BPs in the present study. TCBPA had the highest mean estrogenic activity, contributing $22.4 \%$, followed by BPS (20.2\%), BPA (18.0\%), BPAF (14.7\%), and BPF (9.5\%). DHBP also had weak estrogenic activity, but its contribution to the total was negligible (4.7\%). The $\mathrm{BP}_{2} \mathrm{EQ} \%$ profiles were different from the concentration profiles (Figs. 2 and S3). The most abundant analogue, TBBPA, contributed $37.4 \%$ of the total concentration, but only $3.2 \%$ of the based on $E_{2} E Q \%$ calculations. Higher relative $E_{2} E Q \%$ were observed for the other halogenated BP substitutes (i.e., TCBPA and BPAF), although their percent concentrations were $<4.0 \%$. The EEFs for TCBPA and BPAF $\left(4.30 \times 10^{-4}\right.$ and $\left.1.72 \times 10^{-4}\right)$ were hundreds of times higher than that of TBBPA $\left(4.5 \times 10^{-7}\right.$, Table S4). Our calculation of the estrogenic activity potentials of BP analogues in sludge samples is a preliminary assessment and is subject to several uncertainties. Detailed, peer-reviewed toxicological data concerning the estrogenic activities of various BP analogues are scarce, hindering accurate assessment of the effects of BPs in the environment. For example, TCBPA was reported to have strong estrogenic activity compared to other BP analogues (Kitamura et al., 2005) and thus, we estimated a high relative estrogenic activity for TCBPA. However, relatively weak activities were reported for TBBPA and TMBPA, which are both substituted at the same positions (Kanai et al., 2001). No clear estrogenic activity was found for TBBPA or TCBPA in our MVLN assay. Nevertheless, our results suggest that, although BP analogues such as TBBPA, BPA, BPS, and BPF are predominant in sewage sludge, the biological effects of BPs may be associated with the occurrence and contributions of specific analogues. The toxicological implications of these results warrant further investigation.

\section{Conclusions}

In this study, $8 \mathrm{BP}$ analogues were positively identified in most of the 52 samples from WWTPs, indicating wide-spread presence of BPs in sludge from various parts of China. Similarly high concentrations of BPS and BPF to BPA may indicate extensive use of alternative BPs in certain consumer applications, whereas lower detections of other BP substitutes such as TCBPA, BPAF, BPE, and DHBP indicate some use and/or manufacturing sources. A preliminary in vitro $17 \beta$-estradiol equivalent assessment suggested that the investigated BPs in sludge may have weak estrogenic activity potential, which may mainly arise from specific BP analogues. More detailed research on the behavior and fate of BPs, such as adsorption thermodynamics, biodegradation kinetics, and bioavailability in various environmental compartments are needed and will assist in evaluating the risk of potential adverse effects to human health and the environment.

\section{Acknowledgments}

This work was jointly supported by the National Natural Science Foundation $(21207140,20921063,21077116)$ and the National Basic Research Program of China (2009CB421605). Further support was given by the External Cooperation Program of Chinese Academy of Sciences (GJHZ1202).

\section{Appendix A. Supplementary data}

Supplementary data related to this article can be found at http:// dx.doi.org/10.1016/j.envpol.2013.11.023.

\section{References}

Cacho, J.I., Campillo, N., Vinas, P., Hernandez-Cordoba, M., 2012. Stir bar sorptive extraction coupled to gas chromatography-mass spectrometry for the determination of bisphenols in canned beverages and filling liquids of canned vegetables. J. Chromatogr. A 1247, 146-153.

Cases, V., Alonso, V., Argandoña, V., Rodriguez, M., Prats, D., 2011. Endocrine disrupting compounds: a comparison of removal between conventional activated sludge and membrane bioreactors. Desalination 272, 240-245.

Chen, M.Y., Ike, M., Fujita, M., 2002. Acute toxicity, mutagenicity, and estrogenicity of bisphenol-A and other bisphenols. Environ. Toxicol. 17, 80-86.

Chu, S., Haffner, G.D., Letcher, R.J., 2005. Simultaneous determination of tetrabromobisphenol A, tetrachlorobisphenol A, bisphenol A and other halogenated analogues in sediment and sludge by high performance liquid chromatographyelectrospray tandem mass spectrometry. J. Chromatogr. A 1097, 25-32.

Cirja, M., Ivashechkin, P., Schäffer, A., Corvini, P.F.X., 2008. Factors affecting the removal of organic micropollutants from wastewater in conventional treatment plants (CTP) and membrane bioreactors (MBR). Rev. Environ. Sci. Biotechnol. 7, 61-78.

Clarke, B.O., Smith, S.R., 2011. Review of 'emerging' organic contaminants in biosolids and assessment of international research priorities for the agricultural use of biosolids. Environ. Int. 37, 226-247.

Cobellis, L., Colacurci, N., Trabucco, E., Carpentiero, C., Grumetto, L., 2009. Measurement of bisphenol A and bisphenol B levels in human blood sera from healthy and endometriotic women. Biomed. Chromatogr. 23, 1186-1190.

Cunha, S.C., Almeida, C., Mendes, E., Fernandes, J.O., 2011. Simultaneous determination of bisphenol A and bisphenol B in beverages and powdered infant formula by dispersive liquid-liquid micro-extraction and heart-cutting 
multidimensional gas chromatography-mass spectrometry. Food Addit. Contam. Part A Chem. Anal. Control Expo. Risk Assess. 28, 513-526.

Cunha, S.C., Fernandes, J.O., 2010. Quantification of free and total bisphenol A and bisphenol B in human urine by dispersive liquid-liquid microextraction (DLLME) and heart-cutting multidimensional gas chromatography-mass spectrometry (MD-GC/MS). Talanta 83, 117-125.

Delfosse, V., Grimaldi, M., Pons, J.L., Boulahtouf, A., le Maire, A., Cavailles, V. Labesse, G., Bourguet, W., Balaguer, P., 2012. Structural and mechanistic insights into bisphenols action provide guidelines for risk assessment and discovery of bisphenol A substitutes. Proc. Natl. Acad. Sci. U. S. A. 109, 14930-14935.

Fromme, H., Kuchler, T., Otto, T., Pilz, K., Muller, J., Wenzel, A., 2002. Occurrence of phthalates and bisphenol $\mathrm{A}$ and $\mathrm{F}$ in the environment. Water Res. 36, 14291438.

Fukazawa, H., Hoshino, K., Shiozawa, T., Matsushita, H., Terao, Y., 2001. Identification and quantification of chlorinated bisphenol $\mathrm{A}$ in wastewater from wastepaper recycling plants. Chemosphere 44, 973-979.

Gorga, M., Martinez, E., Ginebreda, A., Eljarrat, E., Barcelo, D., 2013. Determination of PBDEs, HBB, PBEB, DBDPE, HBCD, TBBPA and related compounds in sewage sludge from Catalonia (Spain). Sci. Total Environ. 444, 51-59.

He, M.J., Luo, X.J., Yu, L.H., Liu, J., Zhang, X.L., Chen, S.J., Chen, D., Mai, B.X., 2010. Tetrabromobisphenol-A and hexabromocyclododecane in birds from an ewaste region in South China: influence of diet on diastereoisomer- and enantiomer-specific distribution and trophodynamics. Environ. Sci. Technol. 44, $5748-5754$.

He, Y.J., Chen, W., Zheng, X.Y., Wang, X.N., Huang, X., 2013. Fate and removal of typical pharmaceuticals and personal care products by three different treatment processes. Sci. Total Environ. 447, 248-254.

Kanai, H., Barrett, J.C., Metzler, M., Tsutsui, T., 2001. Cell-transforming activity and estrogenicity of bisphenol-A and 4 of its analogs in mammalian cells. Int. J. Cancer 93, 20-25.

Kitamura, S., Suzuki, T., Sanoh, S., Kohta, R., Jinno, N., Sugihara, K., Yoshihara, S., Fujimoto, N., Watanabe, H., Ohta, S., 2005. Comparative study of the endocrinedisrupting activity of bisphenol A and 19 related compounds. Toxicol. Sci. 84, 249-259.

Konkel, L., 2013. BPA as a mammary carcinogen: early findings reported in rats. Environ. Health Perspect. 121 a284-a284.

Lee, H.B., Peart, T.E., 2002. Organic contaminants in Canadian municipal sewage sludge. Part I. Toxic or endocrine-disrupting phenolic compounds. Water Qual. Res. J. Canada 37, 681-696.

Li, D., Zhou, Z., Qing, D., He, Y., Wu, T., Miao, M., Wang, J., Weng, X., Ferber, J., Herrinton, L., 2010. Occupational exposure to bisphenol-A (BPA) and the risk of self-reported male sexual dysfunction. Human Rep. 25, 519-527.

Liao, C., Liu, F., Alomirah, H., Loi, V.D., Mohd, M.A., Moon, H.B., Nakata, H., Kannan, K., 2012a. Bisphenol S in urine from the United States and seven Asian countries: occurrence and human exposures. Environ. Sci. Technol. 46, 68606866.

Liao, C., Liu, F., Kannan, K., 2012b. Bisphenol S, a new bisphenol analogue, in paper products and currency bills and its association with bisphenol A residues. Environ. Sci. Technol. 46, 6515-6522.

Liao, C., Liu, F., Moon, H.B., Yamashita, N., Yun, S., Kannan, K., 2012c. Bisphenol analogues in sediments from industrialized areas in the United States, Japan, and Korea: spatial and temporal distributions. Environ. Sci. Technol. 46, 1155811565.

Liao, C.Y., Liu, F., Guo, Y., Moon, H.B., Nakata, H., Wu, Q., Kannan, K., 2012d. Occurrence of eight bisphenol analogues in indoor dust from the United States and several Asian countries: implications for human exposure. Environ. Sci. Technol. 46, 9138-9145.
Liu, S., Ying, G.G., Zhao, J.L., Chen, F., Yang, B., Zhou, L.J., Lai, H.J., 2011. Trace analysis of 28 steroids in surface water, wastewater and sludge samples by rapid resolution liquid chromatography-electrospray ionization tandem mass spectrometry. J. Chromatogr. A 1218, 1367-1378.

Morris, S., Allchin, C.R., Zegers, B.N., Haftka, J.J., Boon, J.P., Belpaire, C., Leonards, P.E., Van Leeuwen, S.P., De Boer, J., 2004. Distribution and fate of HBCD and TBBPA brominated flame retardants in North Sea estuaries and aquatic food webs. Environ. Sci. Technol. 38, 5497-5504.

Nyholm, J.R., Grabic, R., Arp, H.P., Moskeland, T., Andersson, P.L., 2013. Environmental occurrence of emerging and legacy brominated flame retardants near suspected sources in Norway. Sci. Total Environ. 443, 307-314.

Oberg, K., Warman, K., Oberg, T., 2002. Distribution and levels of brominated flame retardants in sewage sludge. Chemosphere 48, 805-809.

Pang, G.F., Fan, C.L., Chang, Q.Y., Li, Y., Kang, J., Wang, W.W., Cao, J., Zhao, Y.B., Li, N., Li, Z.Y., Chen, Z.M., Luo, F.J., Lou, Z.Y., 2013. High-throughput analytical techniques for multiresidue, multiclass determination of 653 pesticides and chemical pollutants in tea-part III: evaluation of the cleanup efficiency of an SPE cartridge newly developed for multiresidues in tea. J. AOAC Int. 96, 887896.

Ruan, T., Liu, R., Fu, Q., Wang, T., Wang, Y., Song, S., Wang, P., Teng, M., Jiang, G., 2012. Concentrations and composition profiles of benzotriazole UV stabilizers in municipal sewage sludge in China. Environ. Sci. Technol. 46, 2071-2079.

Saint-Louis, R., Pelletier, E., 2004. LC-ESI-MS-MS method for the analysis of tetrabromobisphenol A in sediment and sewage sludge. Analyst 129, 724-730.

Song, S.J., Ruan, T., Wang, T., Liu, R.Z., Jiang, G.B., 2012. Distribution and preliminary exposure assessment of bisphenol AF (BPAF) in various environmental matrices around a manufacturing plant in China. Environ. Sci. Technol. 46, 13136-13143.

Soto, A.M., Brisken, C., Schaeberle, C., Sonnenschein, C., 2013. Does cancer start in the womb? Altered mammary gland development and predisposition to breast cancer due to in utero exposure to endocrine disruptors. J. Mammary Gland Biol. Neoplasia 18, 199-208.

Sun, J., Liu, J., Liu, Q., Ruan, T., Yu, M., Wang, Y., Wang, T., Jiang, G., 2013. Hydroxylated polybrominated diphenyl ethers (OH-PBDEs) in biosolids from municipal wastewater treatment plants in China. Chemosphere 90, 2388-2395.

Thomsen, C., Janak, K., Lundanes, E., Becher, G., 2001. Determination of phenolic flame-retardants in human plasma using solid-phase extraction and gas chromatography-electron-capture mass spectrometry. J. Chromatogr. B 750, 1 11.

Vinas, P., Campillo, N., Martinez-Castillo, N., Hernandez-Cordoba, M., 2010. Comparison of two derivatization-based methods for solid-phase microextractiongas chromatography-mass spectrometric determination of bisphenol A, bisphenol S and biphenol migrated from food cans. Anal. Bioanal. Chem. 397, 115-125.

Wright, C.T., Paul, D.R., 1997. Gas sorption and transport in UV-irradiated polyarylate copolymers based on tetramethyl bisphenol-A and dihydroxybenzophenone. J. Membr. Sci. 124, 161-174.

Yeo, M., Patisaul, H., Liedtke, W., 2013. Decoding the language of epigenetics during neural development is key for understanding development as well as developmental neurotoxicity. Epigenetics 8, 1128-1132.

Zhao, J., Li, Y., Zhang, C., Zeng, Q., Zhou, Q., 2008. Sorption and degradation of bisphenol A by aerobic activated sludge. J. Hazard. Mater. 155, 305-311.

Zota, A.R., Linderholm, L., Park, J.S., Petreas, M., Guo, T., Privalsky, M.L., Zoeller, R.T., Woodruff, T.J., 2013. Temporal comparison of PBDEs, OH-PBDEs, PCBs, and OHPCBs in the serum of second trimester pregnant women recruited from San Francisco General Hospital, California. Environ. Sci. Technol. 47, 11776-11784. 